\title{
Zur pathologischen Anatomie der centralen und perinuclearen Katarakt.
}

\author{
Von \\ Dr. Eugen v. Hippel, \\ Privatdocenten und erstem Assistenten der Universitats-Augenklinik \\ in Heidelberg. \\ Hierzu Tafel I und II, Fig. 1-4.
}

Nachdem die Untersuchungen ron Deutschmann ${ }^{1}$ ), Beselin ${ }^{9}$ ) und Law ford ${ }^{3}$ ) den Anfang unserer Kenntnisse über die pathologische Anatomie des Schichtstaars begründet hatten, waren es vor allen Schirmer's ${ }^{4}$ ) ausführliche Arbeiten, die das anatomische Substrat der Schichtstaartrübung sicher stellten. Seine Befunde wurden von Hess ${ }^{6}$ ) und Peters ${ }^{6}$ ) in allen wesentlichen Punkten bestätigt. Dass die Trübungszone bedingt wird durch das massenhafte Auftreten kleiner zwischen den Linsenfasern liegender Tröpfchen, die am dichtesten angehäuft sind in einer auf dem Durchschnitt in Gestalt eines ovalen Bandes erscheinenden Zone, dass femer nicht nur diese Zone, sondern

1) v. Graefe's Archiv XXXII. 2., S. 295. 1886.

2) Archiv f. Augenheilkunde. XVIII. S. 71. 1888.

s) Royal London ophth. Hosp. Rep. Vol. XII, P. II, S. 184. 1888.

4) v. Graefe's Archiv XXXV. 3. 1889, v. Graefe's Archiv XXXV1. 1. 1890. v. Graefe's Archiv XXXVII. 4. 1891.

5) y. Graefe's Archiv XXXIX. 1. 1893.

6) ibidem.

v. Graefe's Archiv fitr Ophthalmologie. XLI. 3. 
E. v. Hippel.

auch der Kern von spärlicheren Tröpfchen und grösseren Lücken durchsetzt ist, sowie endlich, dass ein principieller Unterschied zwischen Schicht- und Centralstaar nicht besteht, darf wohl jetzt als sicher gelten. Ferner herrscht Uebereinstimmung darin, dass in den meisten, wenngleich nicht in allen Fällen, eine starke Schrumpfung des Linsensystems nachweisloar ist.

Eine wesentliche principielle Meinungsverschiedenheit besteht nur zwischen Schirmer und Peters bezüglich der Art der Entstehung der Trübungszone. Während Schirmer bekanntlich eine Erkrankung der ganzen Iinse durch eine Ernährungsstörung annimmt, durch welche die periphersten jüngsten Fasern am meisten leiden, glaubt Peters, dass es sich um eine primäre Erkrankung des Kerns handele, durch dessen Schrumpfung erst die übrigen Veränderungen zu Stande kommen. Er greift auch wieder auf die alte Annahme zurück, dass die rhachitischen Krämpfe, die lange anhaltenden Contractionen des Ciliarmuskels zu der Erkrankung der Linse führen, dass demnach die mangelnde Zufuhr normalen Nährmaterials und nicht pathologische Zusammensetzung desselben die Trübung bewirken. Er hält auch für die Naphthalin- und diabetische Katarakt es nicht für erwiesen, dass es sich um Zufuhr pathologischen Nährmaterials handele. Ich möchte auf' diese Controverse nicht weiter eingehen, da meine Untersuchungen kein neues Beweismaterial in dieser Richtung beibringen, anatomische Untersuchungen werden hier überhaupt kaum die definitive Entscheidung liefern. Was die von Peters vorausgesetzte schädliche Wirkung der Ciliarmuskel-Contractionen anlangt, so möchte ich nur zu bedenken geben, dass bei Patienten, die wir Monate lang unter Eserin bez. Pilocarpinwirkung halten, meines Wissens eine schädliche Einwirkung auf die Linse nicht beobachtet worden ist.

Die wichtige Frage, ob ein grosser Procentsatz der Schichtstaare intrauterin entsteht und ob sich aus einem 
angeborenen Totalstaar einmal ein Schichtstaar entwickeln kann, kömte wohl beantwortet werden, wenn sich jemand der Mühe unterzöge, an grossen geburtshilflichen Anstalten die Neugeborenen durch längere Zeiträume zu untersuchen und mit congenitalen Katarakten behaftete Individuen weiter zu verfolgen.

$\mathrm{Ob}$ alle Schicht- und Centralstaare in der gleichen Weise entstehen, dürfte zur Zeit noch nicht zu entscheiden sein. Seine interessante Beobachtung von Schichtstaar beider Augen, wo die Trübung scharf auf die temporale Seite der Linse beschränkt war, sucht Purtscher ${ }^{1}$ ) durch entwickelungsgeschichtliche Störungen zu erklären, für welche die von Hess ${ }^{2}$ ) mitgetheilte Linsenmissbildung bei einem Hühnchen das Verständniss anbahnt.

Der Einfluss der Heredität für die Entstehung verschiedenartiger Staurtrübungen bei Mitgliedern einer $\mathrm{Fa}_{\mathrm{a}}$ milie ist bekannt.

Solche Verhältnisse liegen vor bei den Fällen, die ich hier beschreiben möchte; dieselben wurden in der Universitäts-Augenklinik in Halle beobachtet und operirt, die 4 Linsen wurden mir von meinem Vater freundlichst zur Untersuchung überlassen.

Lina Lippio 32 J. Aufnahme 30. X. 1893. Vor 20 ,ahren Iridektomie an beiden Augen; das Selivermögen war immer mangellaft, soll sich aber im letzten Jalire bedeutend verschlechtert haben. Patientin hat 5 Kinder: 2 davon sind vor 4 Jahren in der Hallenser Klinik wegen Schichtstaar iridektomirt worden, das dritte wurde mit der Mutter in die Klinik aufgenommen und leidet ebenfalls an Staar; das vierte und fünfte sollen gesunde Augen haben, ebenso die Eltern der Patientin.

Status präs. (im Auszug). L. A. Linse gesclırumpft, der Rand im Colobom sichtbar. Die vordersten Corticalsehichten sind klar, dann folgt gegen das Centrum hin eine aus 3 breiten

1) Purtscher, Casuistischer Beitrag zur Lehre des Schichtstaars. Centralblatt f. prakt. Augenheilkunde 1894. Februar.

2) 1. c. 
E. v. Hippel.

Strahlen bestehende intensiv weisse Trubung; jeder dieser Strahlen läuft für sich in ein kleines Dreieck von grellweisser Farbe aus. Vor diesen einzelne strichförmige, radiär gestellte Tribungen, die gegen den Aequator der Linse hinziehen, ohne die Peripherie zu erreichen. Hinter der dreistrahligen Trübung der rorderen Corticalis eine älunliche in der hinteren. Beide sind eingescllossen von einer sclimalen ringförmigen weissgrauen Trübung. Concentrisch zu dieser verläuft in den Aequatorialparthieen eine Anzahl rundlicher ebenfalls weissgrauer Irübungen, die durch durchsichtige Linsensubstanz von einander geschieden sind.

R. A. Im Wesentlichen dieselben Veränderungen wie links, die Trülung ist weiter fortgeschritten, so dass bei enger Pupille gar kein rothes Licht zu erhalten ist. Im Centrum der Linse grellweisse Trubung.

R. $\operatorname{am} 31$. X., L. am 1. XI. modif. Linearextraction mit Benutzung des vorhandenen Coloboms. Linsen treten fast in toto aus; normaler Heilungsverlauf.

2) Iuise Lippig, 3 Jahre. Das Kind macht den Eindruck eines amaurotischen, tappt im Zimmer umher, in der Dämmerung bessere Orientirung.

Starker Nystagmus horizontalis. Im Centrum der geschrumpften Linse intensiv weisse Trübung, die von 2 kreuzförmig gestellten hellen Streifen unterbrochen ist. Umgeben ist die centrale Trübung, die sich nicht durchlenchten lässt, von einer Sehicht rauchig getrubter Corticalis. Dicht unter der Kapsel feine strichförmige Trübungen. Bei enger Pupille kein rothes Licht.

Extraction wie bei der Mutter, normaler Verlauf, Pupillargebiet schwarz.

Die beiden früher iridektomirten Kinder der Frau Lippig konnten, da die Patientin weit entfernt von Halle wohnt, nicht zur Untersuchung bez. Operation kommen.

Wollen wir die Fälle in bestimmte klinisch wohl charakterisirte Gruppen einordnen, so begegnen wir gewissen Schwierigkeiten. Es handelt sich um eine Combination von Centralstaar mit etwas unregelmässigen sehichtstaarartigen Trübungen. Die Falle erinnern etwas an die klinisehen Beobachtungen von Zirm ${ }^{1}$ ) (Fall IV und V) and vor allen Dingen, wie auch die anatomisehe Untersuchung zeigen wird, an den Fall I you Hess"), Ob die

1) Zirm. Klinische Mon.-Bl, f. Augenheilk. 1892. S. 5.

2) $1 . \mathrm{c}$. 
Katarakten angeboren waren, ist mit Sicherheit nicht $\mathrm{zu}$ entscheiden; der Umstand, dass 3 Kinder einer Mutter daran leiden, spricht jedenfalls mit Wahrscheinlichkeit dafür.

Vollkommen stationär sind die Trübungen offenbar nicht gewesen, da bei der Mutter das Sehrermögen im letzten Jalire erheblich abnahn.

Anatomische Untersuchung: Härtung der Linsen in Müller'scher Flüssigkeit und Alcohol. Einbettung in Celloidin. Serienschnitte mit dem Mikxotom. Untersuchung von ungefärbten sowie in Hämatoxylin-Eosin gefärbten Präparaten.

Mutter. Linkes Auge: Meridionalschnitt dureh die Mitte der Linse. Grösster äquatorialer Durehmesser der gehärteten Linse: 6 , sagittaler $3^{1 / 4} \mathrm{~mm}$.

In den im Ganzen normalen peripheren Corticalschichten finden sich einmal Spalten, die Liunstproducte darstellen, da sie vollkommen leer sind und es sich nachweisen lässt, dass die Enden der hier einfach zerrissenen Linsenfasern genar aneinander passen, damn aber noch andere erheblich sehmälere, die mit feinkörniger in Hämatoxylin vielfach blassviolett gefärbter Masse erfüllt sind; ausser diesen kommen noch ziemlich viele sclmale spindelförmige Hohlräume, besonders in den äquatorialen Theilen der Jinse vor.

Ein breiter Spalt durelsetzt auf der einen Seite die Linse entsprechend dem Sternstrahl, hier endigen die Linsenfasern breit and stumpf, der Spalt selbst ist mit körmiger Masse erfüllt.

Nach einwärts von der wenig veränilerten Corticalis folgt eine Schicht, die auf dem Durchschnitt die Form eines ovalen Bandes hat, das in der äquatorialen Zone recht breit, in der vorderen und hinteren Corticalis sehr schmal ist. Es setzt sich zusammen aus grossen, meist ovalen spindelförmigen dicht gedrängten Myelintröpfchen, die mit Hämatoxylin eine etwas dunklere Färbung annehmen. Weiter einwärts folgt eine sehr sclmale, auf dem Durchschnitt ebenfalls ovale Zone, die vorne und hinten mit der eben geschilderten in directer Berührung steht, während sie in der Aequatorialgegend durch eine schmale Schicht annähernd normaler Linsensubstanz davon geschieden ist. Sie besteht aus dichtgedrängten Tröpfchen, die auch mattviolette Färbung annehmen und wie es scheint in einer Reihe schmaler unregelmässiger Spalten gelegen sind. Daneben sind sie auch reihenweise zwischen die Linsenfasern gelagert. Die ganze einwärts von dieser Zone gelegene Linsensubstanz ist durchsetzt von 
E. v. Hippel.

massenhaften etwas gröberen meist nicht gefärbten Tröpfchen, die ganz den Schirmer'schen gleichen. Sie liegen zwischen den Fasern, sind nicht überall in gleicher Menge vorhanden, sondern liegen am dichtesten in der Nähe der gleich zu beschreibenden. Flecken. Ziemlich symmetrisch zu beiden Seiten sowohl in der vorderen wie in der hinteren Corticalis liegen nämlich an je einex circumscripten Stelle solche Tröpfehen in ausserordentlich dichter Anhäufung; đadurch dass sie sich hier mit Hämatoxylin intensiv dunkelviolett färben, erscheinen diese Stellen an gefärbten Präparaten makroskopisch als ca. $1 / 2-3 / 4 \mathrm{~mm}$ grosse Flecken. Es handelt sich hier nicht nur um die ganz kleinen und etwas gröberen Tröpfehen, sondern auch um grössere tropfenartige, stark lichtbrechende Gebilde, die wohl durch Confluiren kleinerer Tropfen entstanden zu denken sind. Endlich sieht man in der Umgebung dieser Flecken theils langgestreckte theils unregelmässig gewundene schmale Spalten, die zum Theil auf der Richtung der Linsenfasern senkrecht stehen, also wohl dureh Schrumpfung im Alkohol entstandene Sprünge sind.

In der einen Hälfte der Linse triffit man in Schnitten, die etwas peripher von der Mitte herstammen, annähernd in der Mitte des Schnittes am Rande einer mit körniger Masse erfüllten. Spalte einen Zug von etwas länglichen Zellen mit dunkelviolett gefürbtem Kern. Die Anordnung der Zellen ist der Art, dass man Anfangs denken konnte, es handele sich um ein Gefäss, doch lehrt die Untersuchung an Serienschnitten, dass dies nicht der Fall ist. An der nämlichen Stelle finden sich in verschiedener Anordnung und wechselnder Menge Zellen in 45 auf einander folgenden Schnitten (von $20-25 \mu$ Dicke). In Schnitten, die schon in die äusserste Peripherie der Linse fallen, sind sie nieht mehr zu finden, in der anderen Hälfte der Linse fehlen sie ebenfalls vollkommen. Nach der Gestalt und Aneinanderlagerung: dieser Zellen handelt es sich wohl um Abkömmlinge der Kapselepithelzellen, doch ist ein directer Zusammenhang mit letzteren nicht zu erweisen, so dass die Bedeutung dieser Zellen nicht vollkommen anfzuklïren ist.

Mutter. (R. A.). Die Gestalt der Linse ist ganz anders, als die des linken Auges; sie ist stärker abgeplattet, dagegen länger als die linke. Maasse: $8: 2^{1 / 2} \mathrm{~mm}$. Makroskopisch sind an durch die Mitte gehenden Meridionalsehnitten bei Hämatoxylin-Eosin-Färbung 3 verschiedene Schichten durch Farbendifferenz zu unterscheiden: Die Peripherie färbt sich rosa, dann folgt nach innen ein nicht vollkommen geschlossener ovaler Ring, der sich 
aus dunkelvioletten Fleekchen zusammensetzt, der centralste Theil der linse ist fast ungefärbt geblieben.

Ausser einigen grösseren leeren Spalten in den äusseren Corticalschichten nahe den Polen, die als Kunstproducte aufzufassen sind, finden sich in der Corticalis sowie an der Grenze von Corticalis und Kem Lücken, die mit körnigen Massen erfüllt sind, welche sich mit Hämatoxylin intensiv violett färben. Eine solche von recht unregelmässiger Gestalt erstreckt sich von der Corticalis aus in der Richtung von vorne nach hinten wie ein Keil in den Kern hinein und dürfte auf den Sternstrahl zu beziehen sein. In den änsseren Corticalschichten in der Nähe der Pole sind die Fasern vielfach durch geronnene Flüssigkeit auseinander gedrängt. Ausserdem liegen in den peripheren Corticalschichten (den vorderen, hinteren sowie äquatorialen) reihenweise Einlagerungen blasser, sich gegenseitig abplattender, zum Theil grosser ovaler Tropfen.

Dann folgt nach dem Centrum lin eine im Ganzen normale Zone, die in ihren innersten Schichten vereinzelte kleine mit violett gefärbten Körnchen erfüllte Hohlräume enthält. Nun folgt nach innen zu ein ovaler Ring, der gebildet wird von massenlaften grossen ovalen Tropfen, die mehr oder weniger stark violett gefärbt sind; sie sind in nur ganz sehwach röthlich gefärbte Linsen-Substanz eingelagert. Zwischen diesen gefärbten findet sich noch eine Anzahl feinster und gröberer farbloser Tröpfchen, die besonders dentlich an ungefärbten und in Glycerin eingelegten Sclmitten hervortreten.

(hne scharfe Grenze geht diese ringförmige Zone in den centralsten Theil der Linse ïber. Hier finden sich einmal wieder jene feinsten farblosen Tröpfchen, ferner nicht sehr reichlich die grösseren violett gefärbten Gebilde, endlich sieht man der Faserrichtung entsprechende ziemlich scharf begrenzte Spalten, deren Inhalt theils Hämatoxylin theils Eosinfärbung angenommen hat. Abgesehen von der verschiedenen Gestalt der Linsen ist also der Befund dem am linken entschieden ähnlich, nur liegt die Zone mit den grossen ovalen Tropfen der Kernregion unmittelbar an und die Violettfärbung ist viel ausgesprochener; die circumscripten Flecken sind hier nicht deutlich ausgeprägt.

Kind. R. A. Die Linse ist sehr stark geschrumpft, Maasse 6:2 mm. Untersuchung an Meridionalschnitten durch die Mitte:

Von dieser Linse scheinen nur die periphersten Theile annähernd normal zu sein, auf der einen Seite ist der Kernbogen sehr deutlich erhalten, die Kerne sind gut gefärbt, anf der anderen 
E. v. Hippel.

Seite muss er im Auge bei der Extraction zurückgeblieben sein. Da wo er erhalten ist, erscheinen die jüngsten Fasern normal, nur auffallend breit und mit etwas welligen Contouren versehen, auf beiden Seiten sind entsprechend dem Alter des Individuums Reste von Kernen in den Fasern ziemlich weit nach dem Centrum der Linse hin zu erkennen. Die nach einwärts anf die mit normalen Kernen versehene Selicht folgende Corticalis ist von sehr zahlreichen meist sehr kleinen Tröpfehen durchsetzt, vereinzelt kommen auch gewölnnliche Myelinkugeln vor. An ungefärbten in Glycerin eingelegten Schnitten sieht diese Zone bei schwacher Vergrösserung wie bestäubt aus. An der Seite, wo der Kermbogen erhalten ist, hat ein Theil der Tröpfehen ganz das Aussehen der von Schirmer bei Schichtstaar bescluiebenen. Einwärts von dieser Zone folgt eine ziemlich schmale, welche dieselben Veränderungen in viel geringerem Grade aufweist. Der centrale Theil der Linse ist von der Peripherie dureh die abweichende Färbung schon makroskopisch zu unterscheiden, die Grundsubstanz ist nämlich durch Hämatoxylin nur ganz matt violett gefärbt worden; die Abgrenzung dieser Kernregion gegen die Corticalis ist unregelmässig und zackig. Von der vorderen Corticalis ist sie abgegrenzt durch einige unregelmässige schmale Spalten, welche theils mit Myelinkugeln, theils mit feinkörnigen, đurch Hämatoxylin blausehwarz gefärbten Massen angefüllt sind. An dem Kern treten bei Hämatoxylinfürbung 3 Stellen durch ihre intensive Färbung besonders hervor, 2 auf dem Durchschnitt henkelförmig ausseliende in den äquatorialen Parthieen des Kerns und eine ganz unregelmässig begrenzte centrale, die der vorderen Corticalis näher als der linteren liegt. Die dunkle Färbung ist an feine und gröbere ansserordentlich dicht aneinander gelagerte Tröpfchen gebunden, die in den äquatorialen Parthieen dem Verlauf der Linsenfasern entsprechend angeordnet sind. In der Grenzschicht zwisehen Kem und Corticalis kommen auch einige grössere spindlige mit blauschwarzen körnigen Massen gefülte Hohlräume vor. Endlich ist der ganze Kern durchsetzt von sehr zahlreichen Tröpfehen, die starke Hämatoxylinfärbung annehmen und am dichtesten in einer Zone liegen, welche ein auf dem Durchsehnitt annähernd ovales Band darstellt.

Kind. L. A. Im Wesentlichen dieselben Verhältnisse wie rechts. Die Schrumpfung ist ebenfalls sehr stark. In der Corticalis finden sich sehr grosse unregelmässige mit geronnener Flüssigkeit erfullte Spalträume. Die Linsenfasern sind grossentheils resorbirt. Stellenweise sind die Linsenfasern kreuzweise 
über die Spalten hinübergespannt. Auffallend ist es, dass der Kern an seinem hinteren Pole eine napfförmige Einsenkung zeigt, deren Concavität nach vorne gerichtet ist. Die betreffende Stelle ist kennthich an der dunkelvioletten Farbe des Inhaltes einer hier befindlichen Spalte. Die Linsenfasern verlaufen hier stark gekrümmt.

Was die Bedeutung der vorgefundenen Veränderungen angeht, so kann man zunächst, wenn man die Krankengeschichte mit den Befunden vergleicht, mit ziemlicher Sicherheit behaupten, dass die schmalen mit feinkörniger Masse gefüllten Spalten in der sonst normalen Corticalis in der linken Linse der Mutter keine Truibung verursacht haben. Die klinisch hier beobachteten zarten perinuclearen Trübungen sind hervorgerufen durch Einlagerung gröberer und feiner tröpfchenartiger Gebilde, die deutlich eine Anordnung in mehreren concentrischen Schichten erkonnen lassen. Freilich sind dieselben nicht scharf gegen einander abgegrenzt, sondern zeigen dadurch allmählichen Uebergang in die an die Trübungszonen angrenzenden Parthieen, dass sich die Tröpfchen hier äusserst spärlich finden. Dass die im Leben grellweiss erscheinenden dreistrahligen Triibungen durch die fleckweise enorme Anhäufung der mit Hämatoxylin so intensiv färbbaren Tröpfchen bedingt sind, kann keinem Zweifel unterliegen. Der ganze Kern zeigt starke Durchsetzung mit kleinen Tröpfchen.

An dem rechten Ange haben die breiten mit durch Hämatoxylin intensiv gefärbten Massen erfüllten Spalten, die von der Corticalis in den Kern hinein sich erstrecken und vielleicht in Beziehung zu dem Sternstrahl stehen, wohl auch im Leben Trübungen verursacht. Die Veränderungen des Kernes sind rechts viel hochgradiger, als links, besonders die Spalten mit den dunkelviolett gefärbten Tropfen sind sehr auffüllig, im Leben war ja auch rechts eine grellweisse centrale Trübung zu sehen. Mit Rücksicht auf den Befund am linken Auge erscheint es sehr wahr- 
E. v. Hippel.

scheinlich, dass die grellweissen Trübungen erzengt werden durch die Massen, welche die intensive Färbung mit Hämatoxylin annehmen. Der ganze Kern der rechten Linse lässt durch sein Verhalten Farbstoffen gegenüber (er nimmt fast gar keine Färbung an) auf eine starke Sklerosirung schliessen. Von einer feinen, durch die Fasercontouren bedingten Streifung sind hier nur Spuren nachweisbar.

Man kann also an beiden Angen von einer Cataracta centralis et perinuclearis sprechen, die perinuclearen Trübungen sind mehrschichtig, die dreistrahligen in vorderer und hinterer Corticalis sind besonders auffällig. Die Trübungen sind verursacht durch Finlagerung verschiedenartiger Tröpfchenbildungen zwischen die Linsenfasern, der Befund schliesst sich denen von Schirmer, Hess und Peters in dieser Richtung an. An beiden Linsen ist eine deutliche Schrumpfung zu erkennen. Der Verlauf des Linsenrandes durch das Colobom sowie die angeführten Maasse beweisen dies. Die Schrumpfung wirkte aber in verschiedener Weise. Während sich links der äquatoriale Durchmesser besonders verkleinerte, wurde die rechte linse stark abgeplattet. Durch diese Art der Schrumpfung mag anch rechts die Fintstehung der aus der Corticalis in den Kern hineinreichenden Spalten zu erklären sein.

Dass der grösste Theil der Veränderungen von früher Kindheit an bestanden hat, unterliegt keinem $Z$ weifel, die Anamnese ergiebt aber auch eine Zunahme der Sehstörung im letzten Jalire; dieselbe kann bedingt sein durch $\mathrm{Zu}$ nahme der vorhandenen Veränderungen sowie secundär durch die fortdauernde Kernschrumpfung, welche zum Auseinanderweichen der Linsenfasern fiihren kann.

Das auffällige Verhalten eines Theils der Tröpfchen gegen Hämatoxylin ist schon einige Male beobachtet worden, so in Schirmer's Fall IV und in dem im Nachtrag (v. Graefe's Archiv XXXVI. 1) beschriebenen Falle. Ferner in Fall 1, 2 u. 4 der Arbeit über Centralstaar 
(v. Graefe's Archiv XXXVII. 4), bei Hess in Fall II und III; man findet dasselbe auch manchmal in tropfenartigen Ausscheidungen bei traumatischer Katarakt.

An den Linsen des Kindes sehen wir eine ausserordentlich starke Schrumpfung, die Staarform ist principiell von der der Mutter nicht verschieden; auch sie ist als Cataracta centralis et perinuclearis zu bezeichnen, die perinuclearen Trübungen zeigen eine weniger regelmässige schichtenweise Anordnung, was wohl durch die Ungleichmässigkeit der Schrumpfung zu erklären ist. Auch hier zeigt es sich, dass die mit Hämatoxylin dunkelviolett gefärbten Parthieen die grellweisse Truibung bedingen.

Sehr auffallend ist besonders in der linken Linse die hochgradige Verflüssigung in der Corticalis, die durch starken Zerfall und nicht allein durch Auseinanderweichen der Linsenfasern $n$ erklären ist. Denn vielfach ragen die Enden der Linsenfasern in diese Hohlräume hinein.

Unter den beschriebenen Fällen von Cataracta centralis hat Fall $I$ von Hess mit den meinigen und unter diesen besonders mit der rechten Linse der Mutter grosse Aehnlichkeit. Ich konnte mich davon an Präparaten, die Herr College Hess mir auf meine Bitte freundlichst zuschickte, ubberzeugen. Ueber das Verhalten des körnigen Tnhaltes der von ihm beschriebenen Hohlräume gegen Hämatoxylin finde ich keine Angabe, die eine Linse war ja auch in toto in Alauncarmin gefärbt worden. Bei der Besprechung seiner Befunde bei Cataracta punctata wirft Hess (pag. 216) die Frage auf, ob die dunkelviolette Färbung des Inhaltes der Hohlräume in seinen Fällen und das Fehlen derselben in den Becker'schen Präparaten von normalen Linsen einen principiellen Unterschied darstellt. Er lässt die Frage offen, da die von Becker benutzte Müller'sche Flüssigkeit die Färbbarkeit hätte beeinträchtigen können. Meine Befunde an in Müller'scher Flüssig- 
keit gehärteten Linsen zeigen, dass ein solcher Einfluss nicht besteht.

In der linken Linse des Kindes fand ich eine ähnliche Einbiegung der Fasern, wie sie Hess schildert, nur mit dem Unterschiede, dass in seinem Falle die Fasern der vorderen Corticalis sich gegen den Kern einsenkten, während in meinem die hintere Kermregion eine solche Einsenkung gegen die hintere Corticalis zeigt.

Ich glaube, diese Befunde sind zu erklären durch Schrumpfung des Kernes, der je nach der Richtung, in welcher die Schrumpfung am stärksten ist, eine verschiedenartige Gestalt annehmen kann. Bei Hess wurde dieselbe biscuitförmig, die vor der Mitte des Kerns gelegenen Linsenfasern wurden nachgezogen; da der Zusammenhang derselben kein so inniger war, dass alle dem Zuge folgen konnten, musste eine Spaltbildung eintreten, die auf dem Durchschnitt die Gestalt eines Dreiecks mit der Basis nach vorne und der Spitze nach hinten haben musste. Eine solche mit geronnener Flüssigkeit gefüllte Spalte ist in den Präparaten von Hess in der That nachzuweisen und auch in seiner Figur 2, wenn auch nicht so schön, wie in manchen Schnitten, kenntlich.

Die beigegebenen Abbildungen verdanke ich der Liebenswürdigkeit des Herrn W. Zangemeister, approb. Arzt, welcher dieselben auf photographischem $W$ ege herstellte.

Erklärung der Abbildungen auf Tafel I u. II.

F i g. 1. Schnitt durch die linke Linse der Mutter (ungefärbtes Glycerin-Präparat, bei auffallendem Lichte photographirt).

Fig. 2. Schnitt durch die rechte Linse der Mutter (Doppelfärbung mit Hämatoxylin - Eosin).

Fig. 3. Schnitt durch die rechte Linse des Kindes (Doppelfärbung mit Hämatoxylin - Eosin).

Fig. 4. Schnitt dureh die linke Linse des Kindes (Doppelfarbung mit Hämatoxylin - Eosin), 

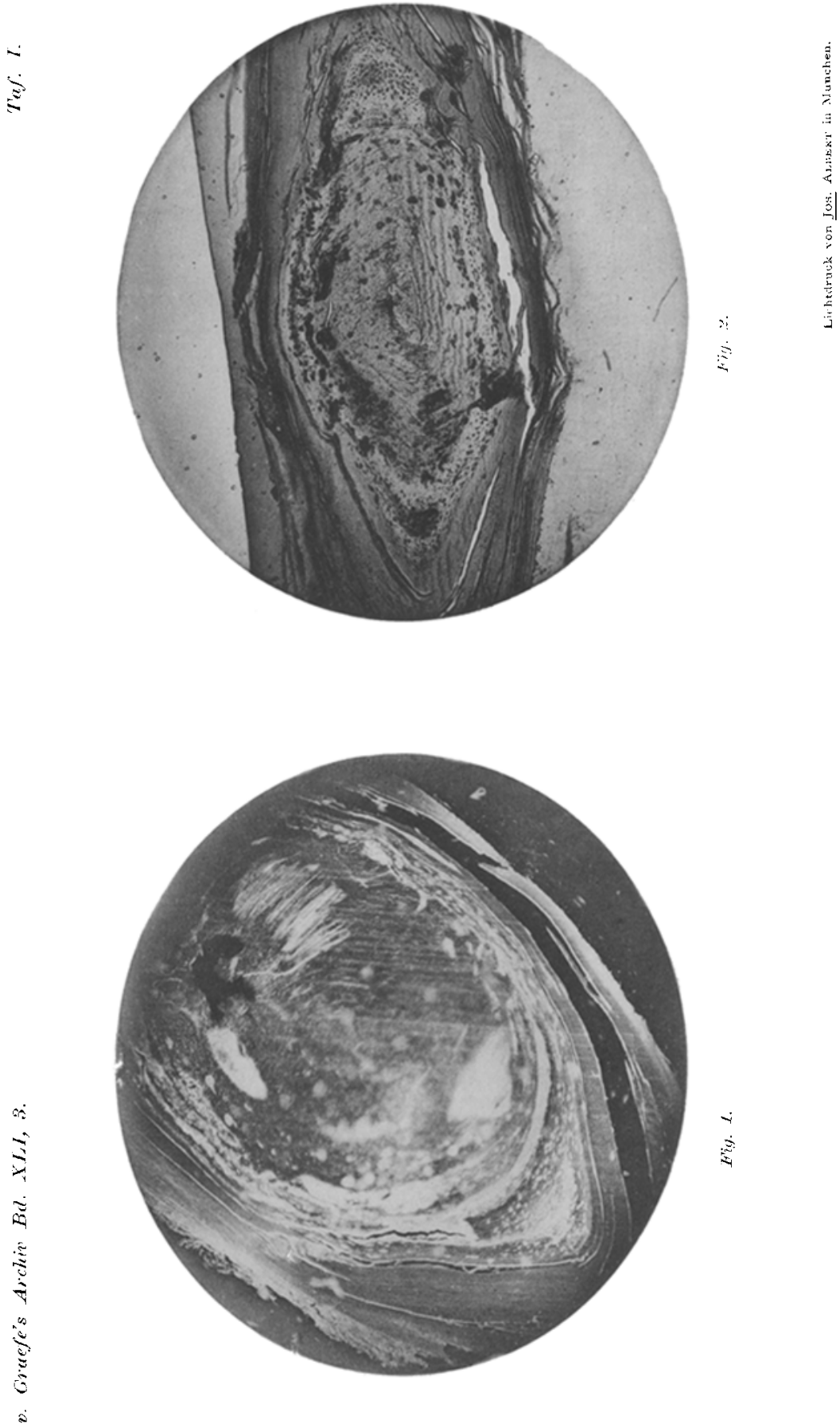


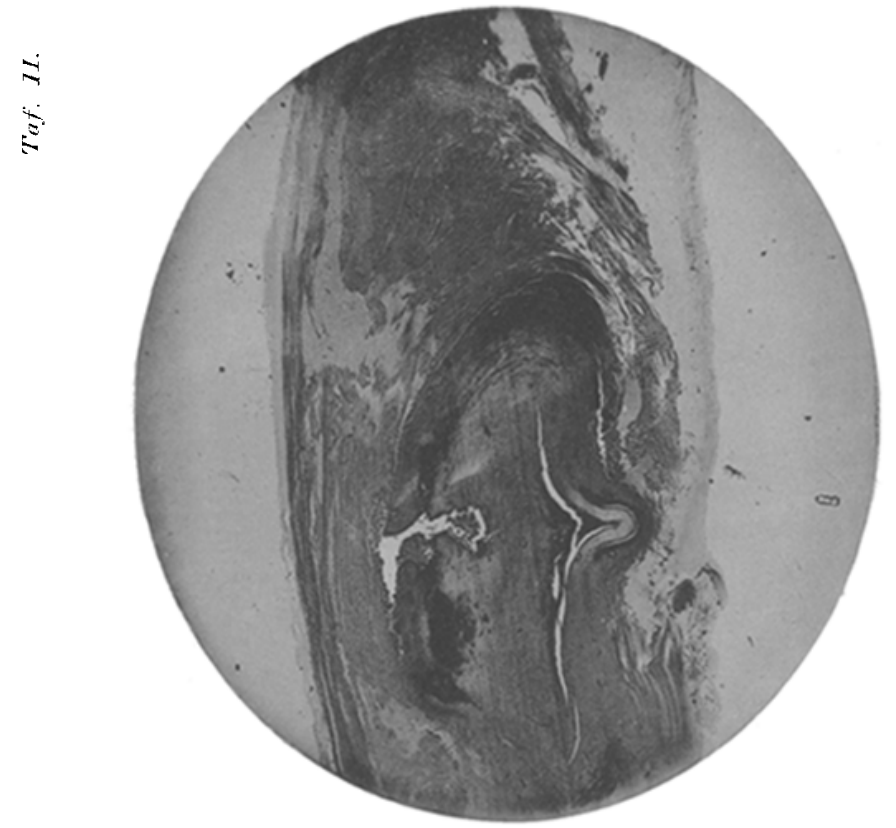

$\dot{\theta}$
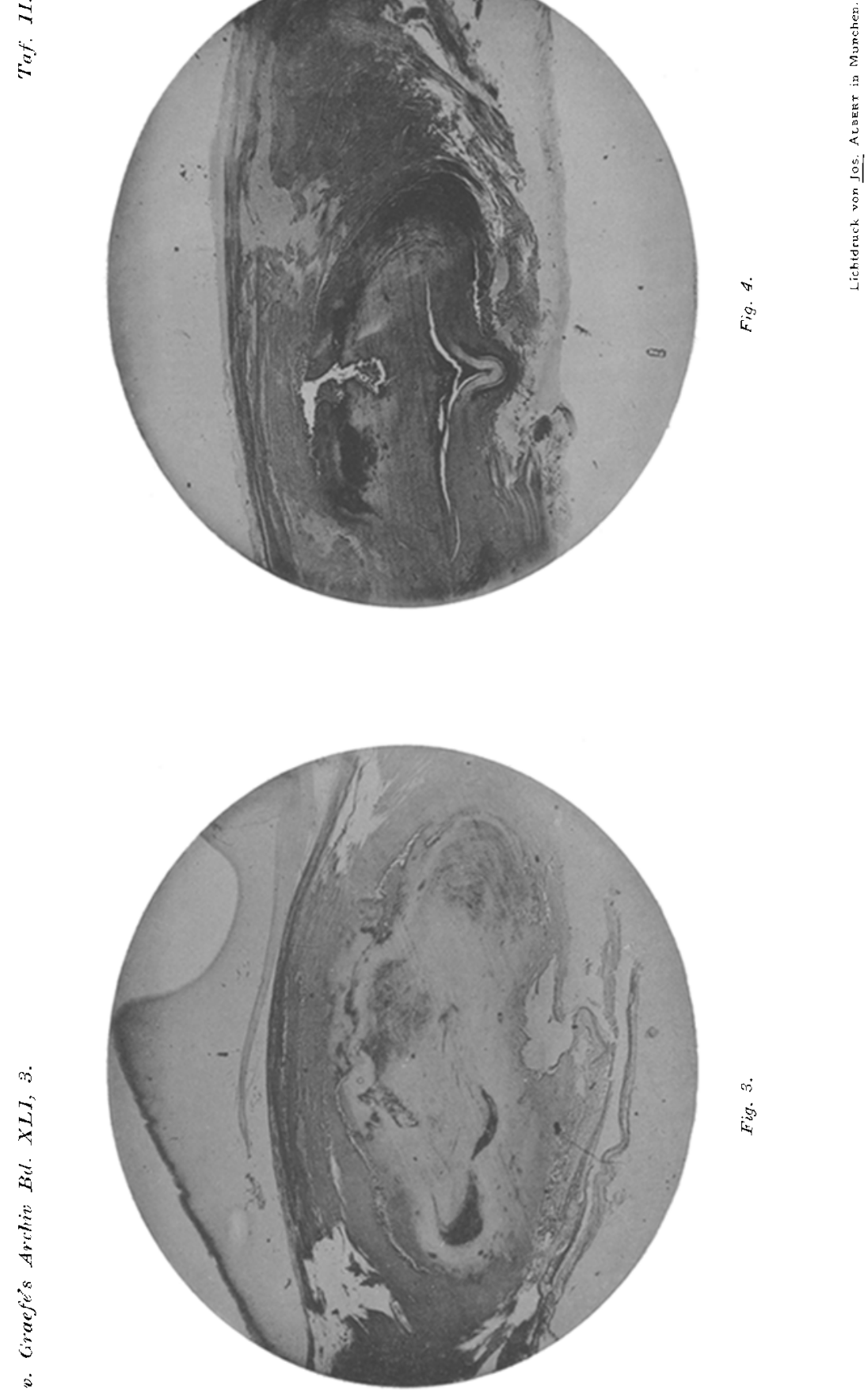

$\dot{s}$ 